\title{
A CRITERION FOR THE UNIQUE SOLVABILITY OF THE POINCARE SPECTRAL PROBLEM IN A CYLINDRICAL DOMAIN FOR ONE CLASS OF MULTIDIMENSIONAL ELLIPTIC EQUATIONS
}

\author{
S. A. Aldashev
}

Institute of Mathematics and Mathematical Modelling Ministry of Education and Science, Almaty, Kazakhstan, E-mail: aldash51@mail.ru

\section{КРИТЕРИЙ ОДНОЗНАЧНОЙ РАЗРЕШИМОСТИ СПЕКТРАЛЬНОЙ ЗАДАЧИ ПУАНКАРЕ В ЦИЛИНДРИЧЕСКОЙ ОБЛАСТИ ДЛЯ ОДНОГО КЛАССА МНОГОМЕРНЫХ ЭЛЛИПТИЧЕСКИХ УРАВНЕНИЙ}

\section{С. А. АЛДАШЕВ}

Институт математики и математического моделирования МОН РК, Алматы, Казахстан, E-mail: aldash51@mail.ru

\begin{abstract}
Two-dimensional spectral problems for elliptic equations are well studied, and their multidimensional analogs, as far as the author knows, are little studied. This is due to the fact that in the case of three or more independent variables there are difficulties of a fundamental nature, since the method of singular integral equations, which is very attractive and convenient, used for two-dimensional problems, cannot be used here because of the lack of any complete theory of multidimensional singular integral equations. The theory of multidimensional spherical functions, on the contrary, has been adequately and fully studied. In the cylindrical domain of Euclidean space, for a single class of multidimensional elliptic equations, the spectral Poincare problem. The solution is sought in the form of an expansion in multidimensional spherical functions. The existence and uniqueness theorems of the solution are proved. Conditions for unique solvability of the problem are obtained, which essentially depend on the height of the cylinder.

KEYWORDs: multidimensional elliptic equation, Poincare spectral problem, multidimensional cylindrical domain, solvability, criterion.
\end{abstract}

АннотАция. Двумерные спектральные задачи для эллиптических уравнений хорошо изучены, а их многомерные аналоги, насколько известно автору, исследованы мало. Это связано с тем, что в случае трех и более независимых переменных возникают трудности принципиального характера, так как весьма привлекательный и удобный метод сингулярных интегральных уравнений, 
применяемый для двумерных задач, здесь не может быть использован из-за отсутствия сколько-нибудь полной теории многомерных сингулярных интегральных уравнений. Теория многомерных сферических функций, напротив, достаточно и полно изучена. В цилиндрической области евклидова пространства для одного класса многомерных эллиптических уравнений рассматривается спектральная задача Пуанкаре. Решение ищется в виде разложения по многомерным сферическим функциям. Доказана теорема существования и единственности решения. Получены условия однозначной разрешимости поставленной задачи, которые существенно зависят от высоты цилиндра.

КлЮчЕВЫЕ СловА: многомерное эллиптическое уравнение, спектральная задача Пуанкаре, многомерная цилиндрическая область, разрешимость, критерий.

\section{ВВеДЕНИЕ}

Корректность краевых задач на плоскости для эллиптических уравнений методом теории аналитических функций комплексного переменного изучена в $[1,2]$.

При исследовании аналогичных вопросов, когда число независимых переменных больше двух, возникают трудности принципиального характера. Весьма привлекательный и удобный метод сингулярных интегральных уравнений теряет свою силу из-за отсутствия сколько-нибудь полной теории многомерных сингулярных интегральных уравнений [3], поэтому в этом направлении мало работ [4-7].

В работе получен критерий однозначной разрешимости спектральной задачи Пуанкаре в цилиндрической области для одного класса многомерных эллиптических уравнений.

\section{1. ПОСТАНОВКА ЗАДАЧИ И РЕЗУЛЬТАТ}

Пусть $\Omega_{\alpha}$ - цилиндрическая область евклидова пространства $E_{m+1}$ точек $\left(x_{1}, \ldots, x_{m}, t\right)$, ограниченная цилиндром $\Gamma=\{(x, t):|x|=1\}$, плоскостями $t=\alpha>0$ и $t=0$, где $|x|$ - длина вектора $x=\left(x_{1}, \ldots, x_{m}\right)$. Части этих поверхностей, образующих границу $\partial \Omega_{\alpha}$ области $\Omega_{\alpha}$ обозначим через $\Gamma_{\alpha}$, $S_{\alpha}, S_{0}$ соответственно.

В области $\Omega_{\alpha}$ рассмотрим взаимно-сопряженные многомерные эллиптические уравнения со спектральным параметром $\gamma$

$$
\begin{gathered}
L u \equiv \Delta_{x} u+u_{t t}+\sum_{i=1}^{m} a_{i}(x, t) u_{x_{i}}+b(x, t) u_{t}+c(x, t) u=\gamma u \\
L^{*} v \equiv \Delta_{x} v+v_{t t}-\sum_{i=1}^{m} a_{i} v_{x_{i}}-b v+d v=\gamma v
\end{gathered}
$$

где $\Delta_{x}$ - оператор Лапласа по переменным $x_{1}, \ldots, x_{m}, m \geq 2$, а 


$$
d(x, t)=c-\sum_{i=1}^{m} a_{i x_{i}}-b_{t} .
$$

В качестве многомерной спектральной задачи Пуанкаре рассмотрим следующую задачу.

Задача 1 . Найти решение уравнения (1) в области $\Omega_{\alpha}$ из класса $C^{1}\left(\bar{\Omega}_{\alpha}\right) \cap$ $C^{2}\left(\Omega_{\alpha}\right)$, удовлетворяющее краевым условиям

$$
\left.u\right|_{S_{\alpha}}=0,\left.\quad u\right|_{\Gamma_{\alpha}}=0,\left.u_{t}\right|_{S_{0}}=0 .
$$

В дальнейшем нам удобно перейти от декартовых координат $x_{1}, \ldots, x_{m}, t$ к сферическим $r, \theta_{1}, \ldots, \theta_{m-1}, t, r \geq 0,0 \leq \theta_{1}<2 \pi, 0 \leq \theta_{i} \leq \pi, i=2,3, \ldots, m-1$.

Пусть $\left\{Y_{n, m}^{k}(\theta)\right\}$ - система линейно независимых сферических функций порядка $n, 1 \leq k \leq k_{n},(m-2) ! n ! k_{n}=(n+m-3) !(2 n+m-2), \theta=$ $\left(\theta_{1}, \ldots, \theta_{m-1}\right), W_{2}^{l}\left(S_{0}\right), l=0,1, \ldots-$ пространства Соболева.

Имеют место [8]

Лемма 1. Пусть $f(r, \theta) \in W_{2}^{l}\left(S_{0}\right)$. Если $l \geq m-1$, то ряд

$$
f(r, \theta)=\sum_{n=0}^{\infty} \sum_{k=1}^{k_{n}} f_{n}^{k}(r) Y_{n, m}^{k}(\theta),
$$

а также рлдь, полученные из него дифферениированием порядка $p \leq l-$ $m+1$, сходлтся абсолютно и равномерно.

Лемма 2. Для того, чтобъ $f(r, \theta) \in W_{2}^{l}\left(S_{0}\right)$, необходимо и достаточно, чтобы коэффичиенты ряда (3) удовлетворяли неравенствам

$$
\left|f_{0}^{1}(r)\right| \leq c_{1}, \sum_{n=1}^{\infty} \sum_{k=1}^{k_{n}} n^{2 l}\left|f_{n}^{k}(r)\right|^{2} \leq c_{2}, \quad c_{1}, c_{2}=\text { const. }
$$

Через $\tilde{a}_{i n}^{k}(r, t), a_{i n}^{k}(r, t), \tilde{b}_{n}^{k}(r, t), \tilde{c}_{n}^{k}(r, t), \tilde{d}_{n}^{k}(r, t), \rho_{n}^{k}$, обозначим коэффициенты разложения ряда (3), соответственно функций $a_{i}(r, \theta, t) \rho(\theta), a_{i} \frac{x_{i}}{r} \rho$, $b(r, \theta, t) \rho, c(r, \theta, t) \rho, d(r, \theta, t) \rho, \rho(\theta), i=1, \ldots, m$, причем $\rho(\theta) \in C^{\infty}(H), H-$ единичная сфера в $E_{m}$.

Пусть

$$
\begin{gathered}
a_{i}(r, \theta, t), b(r, \theta, t), c(r, \theta, t) \in W_{2}^{l}\left(\Omega_{\alpha}\right) \subset C\left(\bar{\Omega}_{\alpha}\right), l \geq m+1, i=1, \ldots, m . \\
b(r, \theta, 0)=0, \forall(r, \theta) \in S_{0}, \quad c(r, \theta, t)-\gamma \leq 0, \forall(r, \theta, t) \in \Omega_{\alpha} .
\end{gathered}
$$

Тогда справедлива

Теорема 1. 1) Если $\gamma \geq-\mu_{s, n}^{2}$, то задача 1 имеет только нулевое решение; 2) При $\gamma<-\mu_{s, n}^{2}$, задача 1 имеет только тривиальное решение тогда и только тогда, когда

$$
\cos \alpha \sqrt{\left|\gamma+\mu_{s, n}^{2}\right|} \neq 0, s=1,2, \ldots
$$

где $\mu_{s, n}-$ положительные нули функиий Бесселя первого рода $J_{n+\frac{(m-2)}{2}}(z)$, $n=0,1, \ldots$ 


\section{2. РАЗРЕШИМОСТЬ ЗАДАЧИ 1}

В сферических координатах уравнения (1) имеет вид

$$
\begin{aligned}
L u \equiv u_{r r}+ & \frac{m-1}{r} u_{r}-\frac{1}{r^{2}} \delta u+u_{t t}+\sum_{i=1}^{m} a_{i}(r, \theta, t) u_{x_{i}}+ \\
& +b(r, \theta, t) u_{t}+c(r, \theta, t) u=\gamma u
\end{aligned}
$$

где

$$
\begin{aligned}
\delta \equiv-\sum_{j=1}^{m-1} \frac{1}{g_{j} \sin ^{m-j-1} \theta_{j}} \frac{\partial}{\partial \theta_{j}}\left(\sin ^{m-j-1} \frac{\partial}{\partial \theta_{j}}\right), \\
g_{1}=1, g_{j}=\left(\sin \theta_{1}, \ldots, \sin \theta_{j-1}\right)^{2}, j>1 .
\end{aligned}
$$

Известно [8], что спектр оператора $\delta$ состоит из собственных чисел $\lambda_{n}=$ $n(n+m--2), n=0,1, \ldots$, каждому из которых соответствует $k_{n}$ ортонормированных собственных функций $Y_{n, m}^{k}(\theta)$.

Искомое решение задачи 1 будем искать в виде

$$
u(r, \theta, t)=\sum_{n=0}^{\infty} \sum_{k=1}^{k_{n}} \bar{u}_{n}^{k}(r, t) Y_{n, m}^{k}(\theta),
$$

где $\bar{u}_{n}^{k}(r, t)-$ функции, подлежащие определению.

Подставив (6) в (5), умножив полученное выражение на $\rho(\theta) \neq 0$ и проинтегрировав по единичной сфере $H$, для $\bar{u}_{n}^{k}$ получим $[5,7]$

$$
\begin{gathered}
\rho_{0}^{1} \bar{u}_{0 r r}^{1}+\rho_{0}^{1} \bar{u}_{0 t t}^{1}+\left(\frac{m-1}{r} \rho_{0}^{1}+\sum_{i=1}^{m} a_{i 0}^{1}\right) \bar{u}_{0 r}^{1}+\tilde{b}_{0}^{1} \bar{u}_{0 t}^{1}+\tilde{c}_{0}^{1} \bar{u}_{0}^{1}-\gamma \rho_{0}^{1} \bar{u}_{0}^{1}+ \\
+\sum_{n=1}^{\infty} \sum_{k=1}^{k_{n}}\left\{\rho_{n}^{k} \bar{u}_{n r r}^{k}+\rho_{n}^{k} \bar{u}_{n t t}^{k}+\left(\frac{m-1}{r} \rho_{n}^{k}+\sum_{i=1}^{m} a_{i n}^{k}\right) \bar{u}_{n r}^{k}+\right. \\
\left.+\tilde{b}_{n}^{k} \bar{u}_{n t}^{k}+\left[\tilde{c}_{n}^{k}-\lambda_{n} \frac{\rho_{n}^{k}}{r^{2}}+\sum_{i=1}^{m}\left(\tilde{a}_{i n-1}^{k}-(n-1) a_{i n}^{k}\right)\right] \bar{u}_{n}^{k}-\gamma \rho_{n}^{k} \bar{u}_{n}^{k}\right\}=0 .
\end{gathered}
$$

Теперь рассмотрим бесконечную систему дифференциальных уравнений

$$
\begin{gathered}
\rho_{0}^{1} \bar{u}_{0 r r}^{1}+\rho_{0}^{1} \bar{u}_{0 t t}^{1}+\frac{m-1}{r} \rho_{0}^{1} \bar{u}_{0 r}^{1}=\gamma \rho_{0}^{1} \bar{u}_{0}^{1}, \\
\rho_{1}^{k} \bar{u}_{1 r r}^{k}+\rho_{1}^{k} \bar{u}_{1 t t}^{k}+\frac{m-1}{r} \rho_{1}^{k} \bar{u}_{1 r}^{k}-\frac{\lambda_{1}}{r^{2}} \rho_{1}^{k} \bar{u}_{1}^{k}= \\
=\gamma \rho_{1}^{k} \bar{u}_{1}^{k}-\frac{1}{k_{1}}\left(\sum_{i=1}^{m} a_{i 0}^{1} \bar{u}_{0 r}^{1}+\tilde{b}_{0}^{1} \bar{u}_{0 t}^{1}+\tilde{c}_{0}^{1} \bar{u}_{0}^{1}\right), \quad n=1, \quad k=\overline{1, k_{1}}, \\
\rho_{n}^{k} \bar{u}_{n r r}^{k}+\rho_{n}^{k} \bar{u}_{n t t}^{k}+\frac{m-1}{r} \rho_{n}^{k} \bar{u}_{n r}^{k}-\frac{\lambda_{n}}{r^{2}} \rho_{n}^{k} \bar{u}_{n}^{k}= \\
=\gamma \rho_{n}^{k} \bar{u}_{n}^{k}-\frac{1}{k_{n}} \sum_{k=1}^{k_{n-1}}\left\{\sum_{i=1}^{m} a_{i n-1}^{k} \bar{u}_{n-1 r}^{k}++\tilde{b}_{n-1}^{k} \bar{u}_{n-1 t}^{k}+\right.
\end{gathered}
$$




$$
\left.+\left[\tilde{c}_{n-1}^{k}-\sum_{i=1}^{m}\left(\tilde{a}_{i n-2}^{k}-(n-1) a_{n-1}^{k}\right)\right] \bar{u}_{n-1}^{k}\right\}, k=\overline{1, k_{n}}, \quad n=2,3, \ldots
$$

Суммируя уравнение (9) от 1 до $k_{1}$, а уравнение (10) от 1 до $k_{n}$, а затем сложив полученные выражения вместе с (8), приходим к уравнению (7).

Отсюда следует, что если $\left\{\bar{u}_{n}^{k}\right\}, k=\overline{1, k_{n}}, n=0,1, \ldots$ - решение системы (8)-(10), то оно является решением уравнения (7).

Нетрудно заметить, что каждое уравнение системы (8)-(10) можно представить в виде

$$
\bar{u}_{n r r}^{k}+\bar{u}_{n t t}^{k}+\frac{m-1}{r} \bar{u}_{n r}^{k}-\frac{\lambda_{n}}{r^{2}} \bar{u}_{n}^{k}=\gamma \bar{u}_{n}^{k}+\bar{f}_{n}^{k}(r, t),
$$

где $\bar{f}_{n}^{k}(r, t)$ определяются из предыдущих уравнений этой системы, при этом $\bar{f}_{0}^{1}(r, t) \equiv 0$.

Далее, из краевого условия (2) в силу (6),будем иметь

$$
\bar{u}_{n}^{k}(r, \alpha)=0, \bar{u}_{n}^{k}(1, t)=0, \bar{u}_{n t}^{k}(r, 0)=0, k=\overline{1, k_{n}}, \quad n=0,1, \ldots
$$

Из (11), (12) произведя замену $\bar{u}_{n}^{k}(r, t)=r^{\frac{(1-m)}{2}} u_{n}^{k}(r, t)$, получим

$$
\begin{gathered}
L u_{n}^{k} \equiv \bar{u}_{n r r}^{k}+u_{n t t}^{k}+\frac{\bar{\lambda}_{n}}{r^{2}} u_{n}^{k}=\gamma u_{n}^{k}+f_{n}^{k}(r, t), \\
u_{n}^{k}(r, \alpha)=0, \quad u_{n}^{k}(1, t)=0, \quad u_{n t}^{k}(r, 0)=0, \quad k=\overline{1, k_{n}}, \quad n=0,1, \ldots, \\
\bar{\lambda}_{n}=\frac{(m-1)(3-m)-4 \lambda_{n}}{4}, \quad f_{n}^{k}(r, t)=r^{\frac{(m-1)}{2}} \bar{f}_{n}^{k}(r, t) .
\end{gathered}
$$

Решение задачи (13), (14) будем искать в виде

$$
u_{n}^{k}(r, t)=\sum_{s=1}^{\infty} R_{s}(r) T_{s}(t)
$$

при этом пусть

$$
f_{n}^{k}(r, t)=\sum_{s=1}^{\infty} a_{n s}^{k}(t) R_{s}(r)
$$

Подставляя (15) в (13), (14), с учетом (16), получим

$$
\begin{gathered}
R_{s r r}+\frac{\bar{\lambda}_{n}}{r^{2}} R_{s}+(\mu-\gamma) R_{s}=0,0<r<1, \\
R_{s}(1)=0, \quad\left|R_{s}(0)\right|<\infty, \\
T_{s t t}-\mu T_{s}(t)=-a_{n s}^{k}(t), 0<t<\alpha, \\
T_{s}(\alpha)=0, T_{s t}(0)=0 .
\end{gathered}
$$

Ограниченным решением задачи (17), (18) является [9]

$$
R_{s}(r)=\sqrt{r} J_{\nu}\left(\mu_{s, n} r\right), 0<r<1,
$$

где $\nu=n+\frac{(m-2)}{2}, \mu=\gamma+\mu_{s, n}^{2}$. 
Общее решение уравнения (20) представимо в виде [9]

$$
T_{s, n}(t)=\left\{\begin{array}{l}
c_{1 s} \operatorname{ch} t \sqrt{\mu}+c_{2 s} \operatorname{sh} t \sqrt{\mu}+\frac{\operatorname{ch} \sqrt{\mu} t}{\sqrt{\mu}} \int_{0}^{t} a_{n s}^{k}(\xi) \operatorname{sh} \sqrt{\mu} \xi d \xi- \\
-\frac{\operatorname{sh} \sqrt{\mu} t}{\sqrt{\mu}} \int_{0}^{t} a_{n s}^{k} \operatorname{ch} \sqrt{\mu} \xi d \xi, \mu>0, \\
c_{1 s}+c_{2 s} t-\int_{0}^{t} a_{n s}^{k}(\xi)(t-\xi) d \xi, \mu=0, \\
c_{1 s} \cos t \sqrt{|\mu|}+c_{2 s} \sin t \sqrt{|\mu|}+ \\
+\frac{\cos \sqrt{|\mu|} t}{\sqrt{|\mu|}} \int_{0}^{t} a_{n s}^{k}(\xi) \sin \sqrt{|\mu|} \xi d \xi- \\
-\frac{\sin \sqrt{|\mu|} t}{\sqrt{|\mu|}} \int_{0}^{t} a_{n s}^{k}(\xi) \cos \sqrt{|\mu|} \xi d \xi, \mu<0,
\end{array}\right.
$$

где $c_{1 s}, c_{2 s}$ - произвольные постоянные. Удовлетворив условие (20) будем иметь

$$
0=\left\{\begin{array}{l}
c_{1 s} \operatorname{ch} \alpha \sqrt{\mu}+\frac{\operatorname{ch} \alpha \sqrt{\mu}}{\sqrt{\mu}} \int_{0}^{\alpha} a_{n s}^{k}(\xi) \operatorname{sh} \sqrt{\mu} \xi d \xi- \\
-\frac{\operatorname{sh} \alpha \sqrt{\mu}}{\sqrt{\mu}} \int_{0}^{\alpha} a_{n s}^{k}(\xi) \operatorname{ch} \sqrt{\mu} \xi d \xi, \mu>0, \\
c_{1 s}-\int_{0}^{\alpha} a_{n s}^{k}(\xi)(\alpha-\xi) d \xi, \mu=0, \\
c_{1 s} \cos \alpha \sqrt{|\mu|}+\frac{\cos \alpha \sqrt{|\mu|}}{\sqrt{|\mu|}} \int_{0}^{\alpha} a_{n s}^{k}(\xi) \sin \sqrt{|\mu|} \xi d \xi- \\
-\frac{\sin \alpha \sqrt{|\mu|}}{\sqrt{|\mu|}} \int_{0}^{\alpha} a_{n s}^{k}(\xi) \cos \sqrt{|\mu|} \xi d \xi, \mu<0 .
\end{array}\right.
$$

Подставляя (21) в (16) получим

$$
r^{-\frac{1}{2}} f_{n}^{k}(r, t)=\sum_{s=1}^{\infty} a_{n s}^{k}(t) J_{\nu}\left(\mu_{s, n} r\right) .
$$

Ряд (24) - разложение в ряд Фурье-Бесселя [10],если

$$
a_{n s}^{k}(t)=\frac{2}{\left[J_{\nu+1}\left(\mu_{s, n}\right)\right]^{2}} \int_{0}^{1} \sqrt{\xi} f_{n}^{k}(\xi, t) J_{\nu}\left(\mu_{s, n} \xi\right) d \xi,
$$

где $\mu_{s, n}, s=1,2, \ldots$ - положительные нули функций Бесселя $J_{\nu}(z)$ расположенные в порядке возрастания их величины.

Из (21), (22), (23) найдем решение задачи (13), (14)

$$
u_{n}^{k}(r, t)=\sum_{s=1}^{\infty} \sqrt{r} T_{s, n}(t) J_{\nu}\left(\mu_{s, n} r\right),
$$

где $a_{n s}^{k}(t)$ находится из (25). 
Следовательно, сначала решив задачу (8), (12) $(n=0)$, а затем (9), (12) $(n=1)$ и т. д. найдем последовательно все $u_{n}^{k}(r, t)$ из $(26), k=\overline{1, k_{n}}, n=$ $0,1, \ldots$

Итак, в области $\Omega_{\alpha}$, имеет место

$$
\int_{H} \rho(\theta)(L-\gamma) u d H=0 .
$$

Пусть $f(r, \theta, t)=R(r) \rho(\theta) T(t)$, причем $R(r) \in V_{0}, V_{0}$ - плотна в $L_{2}((0,1))$, $\rho(\theta) \in C^{\infty}(H)$ - плотна в $L_{2}(H)$, а $T(t) \in V_{1}, V_{1}-$ плотна в $L_{2}((0, \alpha))$. Tогда $f(r, \theta, t) \in V, V=V_{0} \otimes H \otimes V_{1}-$ плотна в $L_{2}\left(\Omega_{\alpha}\right)$ [11].

Отсюда и из (27), следует, что

$$
\int_{\Omega_{\alpha}} f(r, \theta, t)(L-\gamma) u d \Omega_{\alpha}=0
$$

и

$$
L u=\gamma u, \quad \forall(r, \theta, t) \in \Omega_{\alpha} .
$$

Таким образом, решением задачи 1 является функция

$$
u(r, \theta, t)=\sum_{n=0}^{\infty} \sum_{k=1}^{k_{n}} \sum_{s=1}^{\infty} r^{\frac{(2-m)}{2}} T_{s, n}(t) J_{n+\frac{(m-2)}{2}}\left(\mu_{s, n} r\right) Y_{n, m}^{k}(\theta),
$$

где $T_{s, n}(t)$ определяется из $(22)$.

Из $(4),(23)$ следует, что $c_{1 s}=0$, при $\mu \geq 0$ и для $\mu<0, c_{1 s}=0$, если выполняется условие (4). Следовательно, из (25), (22), (26) следует, что $T_{s, n}(t)=0$ и $u_{n}^{k}(r, t)=a_{n s}^{k}(t)=0, s=\overline{1, k_{n}}, n=0,1, \ldots$

Далее, из (28) в свою очередь получим $u=0$ в $\Omega_{\alpha}$.

Пусть теперь условие (4) нарушено, хотя бы для одного $s=l$.

Тогда, если решение задачи 1 будем искать в виде (6), то приходим к краевой задаче (13), (14).

В силу (22), (23) её решением является функция

$$
\begin{aligned}
& u_{n}^{k}(r, t)= \\
& =\sqrt{r}\left[\cos t \sqrt{\left|\gamma+\mu_{l, n}^{2}\right|}+\frac{\cos t \sqrt{\left|\gamma+\mu_{l, n}^{2}\right|}}{\sqrt{\left|\gamma+\mu_{l, n}^{2}\right|}} \int_{0}^{t} a_{n l}(\xi) \sin \xi \sqrt{\left|\gamma+\mu_{l, n}^{2}\right|} d \xi-\right. \\
& \left.\quad-\frac{\sin t \sqrt{\left|\gamma+\mu_{l, n}^{2}\right|}}{\sqrt{\left|\gamma+\mu_{l, n}^{2}\right|}} \int_{0}^{t} a_{n l}(\xi) \cos \xi \sqrt{\left|\gamma+\mu_{l, n}^{2}\right|} d \xi\right] J_{n+\frac{(m-2)}{2}}\left(\mu_{l, n} r\right) .
\end{aligned}
$$

Следовательно, нетривиальные решения задачи 1 записываются в виде ряда

$$
u(r, \theta, t)=\sum_{n=0}^{\infty} \sum_{k=1}^{k_{n}} n^{-p} r^{\frac{(1-m)}{2}} u_{n}^{k}(r, t) Y_{n, m}^{k}(\theta) .
$$


Учитывая формулу [10]

$$
2 J_{\nu}^{\prime}(z)=J_{\nu-1}(z)-J_{\nu+1}(z),
$$

а также оценки $[8,12]\left(c_{1}, c_{2}=\right.$ const $\left., j=\overline{1, m-1}, q=0,1, \ldots\right)$

$$
\begin{gathered}
J_{\nu}(z)=\sqrt{\frac{2}{\pi z}} \cos \left(z-\frac{\pi}{2} \nu-\frac{\pi}{4}\right)+0\left(\frac{1}{z^{3 / 2}}\right), \nu \geq 0, \\
\left|k_{n}\right| \leq c_{1} n^{m-2}, \quad\left|\frac{\partial^{q}}{\partial \theta_{j}^{q}} Y_{n, m}^{k}(\theta)\right| \leq c_{2} n^{\frac{m}{2}-1+q},
\end{gathered}
$$

а также леммы 1,2 , ограничения на коэффициенты уравнения (1), как в [7], можно доказать, что если $p>\frac{3 m}{2}$, то функция (29) принадлежит искомому классу $C^{1}\left(\bar{\Omega}_{\alpha}\right) \cap C^{2}\left(\Omega_{\alpha}\right)$.

Разрешимость задачи 1 установлено.

\section{3. ЕДИНСТВЕННОСТЬ РЕШЕНИЯ ЗАДАЧИ 1}

Сначала построим решение краевой задачи для уравнения $\left(1^{*}\right)$ с данными

$$
\left.v\right|_{S_{\alpha} \cup \Gamma_{\alpha}}=0,\left.v_{t}\right|_{S_{0}}=\nu(r, \theta)=\bar{\nu}_{n}^{k}(r) Y_{n, m}^{k}(\theta), \quad k=\overline{1, k_{n}}, n=0,1, \ldots,
$$

где $\bar{\nu}_{n}^{k}(r) \in G, G$ - множество функций $\nu(r)$ из класса $C([0,1]) \cap C^{1}((0,1))$. Множество $G$ плотно всюду в $L_{2}((0,1))$ [11]. Решение задачи $\left(1^{*}\right),(31)$ будем искать в виде $(6)$, где функции $\bar{v}_{n}^{k}(r, t)$ будут определены ниже. Тогда, аналогично п. 2 , функции $\bar{v}_{n}^{k}(r, t)$ удовлетворяют систему уравнений (8)(10), где $\tilde{a}_{i n}^{k}, a_{i n}^{k}, \tilde{b}_{n}^{k}$, заменены на $-\tilde{a}_{i n}^{k},-a_{i n}^{k},-\tilde{b}_{n}^{k}$, а $\tilde{c}_{n}^{k}$ на $\tilde{d}_{n}^{k}, i=1, \ldots, m$, $k=\overline{1, k_{n}}, n=0,1, \ldots$

Далее, из краевого условия (31), в силу (6), получим

$$
\bar{v}_{n}^{k}(r, \beta)=\bar{v}_{n}^{k}(1, t)=0, \quad \bar{v}_{n t}^{k}(r, 0)=\bar{\nu}_{n}^{k}(r), \quad k=\overline{1, k_{n}}, \quad n=0,1, \ldots .
$$

Как ранее замечено, каждое уравнение системы (8)-(10) представимо в виде (11).

Далее, задача (11), (32), решается, аналогично, как решалась задача (13), (14) из п. 2.

Таким образом, решение задачи $\left(1^{*}\right),(31)$ в виде ряда $(28)$ построено и в силу (30), как показано в [9], принадлежит классу $C^{1}\left(\bar{\Omega}_{\alpha}\right) \cap C^{2}\left(\Omega_{\alpha}\right)$.

Из определения сопряженных операторов $L, L^{*}[13]$

$$
v L u-u L^{*} v=-v P(u)+u P(v)-u v Q,
$$

где

$$
\begin{gathered}
P(u)=\sum_{i=1}^{m} u_{x_{i}} \cos \left(N^{\perp}, x_{i}\right)+u_{t} \cos \left(N^{\perp}, t\right), \\
Q=\sum_{i=1}^{m} a_{i} \cos \left(N^{\perp}, x_{i}\right)-b \cos \left(N^{\perp}, t\right),
\end{gathered}
$$


а $N^{\perp}$ - внутренняя нормаль к границе $\partial \Omega_{\alpha}$, по формуле Грина имеем

$$
\int_{\Omega_{\alpha}}\left(v L u-u L^{*} v\right) d \Omega_{\alpha}=\int_{\partial \Omega_{\alpha}}\left[\left(v \frac{\partial u}{\partial N}-u \frac{\partial v}{\partial N}\right) M+u v Q\right] d s,
$$

где

$$
\begin{aligned}
\frac{\partial}{\partial N} & =\sum_{i=1}^{m} \cos \left(N^{\perp}, x_{i}\right)+\cos \left(N^{\perp}, t\right) \frac{\partial}{\partial t}, \\
M^{2} & =\sum_{i=1}^{m} \cos ^{2}\left(N^{\perp}, x_{i}\right)+\cos ^{2}\left(N^{\perp}, t\right) .
\end{aligned}
$$

Из (33), принимая во внимание граничные условия (2) и условия (31) получим

$$
\int_{S_{0}} \nu(r, \theta) u(r, \theta, 0) d s=0 .
$$

Поскольку линейная оболочка системы функций $\left\{\bar{\nu}_{n}^{k}(r) Y_{n, m}^{k}(\theta)\right\}$ плотна в $L_{2}\left(S_{0}\right)$ [11], то из $(34)$ заключаем, что $u(r, \theta, 0)=0, \forall(r, \theta) \in S_{0}$.

Следовательно, в силу единственности решения задачи Дирихле $[2,3]$

$$
L u=\gamma u,\left.u\right|_{S_{0} \cup \Gamma_{\alpha} \cup S_{\alpha}}=0,
$$

будем иметь $u=0$ в $\bar{\Omega}_{\alpha}$.

Таким образом, единственность решения задачи 1 показано.

Теорема 1 доказана полностью.

\section{ЛитератУРА}

1. Bitsadze A. Equations of mixed type. Moscow: Izd. AN USSR, 1959. (in Russian)

2. Bitsadze A. Boundary value problems for elliptic second order equations. Moscow: Nauka, 1966. (in Russian)

3. Bitsadze A. Some classes of equations in partial derivatives. Moscow: Science, 1981. (in Russian)

4. Aldashev S. A. The correctness of the Dirichlet problem in a cylindrical region for the multidimensional Laplace equation. Izvestia Saratovsky University. Novaya seriya, ser. mat., mech., inf. 2012. Vol. 12. No. 3. P. 3-7. (in Russian)

5. Aldashev S. A. The correctness of the Dirichlet problem in cylindrical region for one class of multidimensional elliptic equations. Bulletin of the NSU, ser., mat., mech., inf. 2012. Vol. 12. Issue 1. P. 7-13. (in Russian)

6. Aldashev S. A. The correctness of the Poincare problem in a cylindrical domain for the multidimensional Laplace equation. News of the National Academy of Sciences of Kazakhstan, ser. Physics and Mathematics. 2014. No. 3. P. 62-67. (in Russian)

7. Aldashev S. A. The correctness of the Poincare problem in cylindrical area for one class in multidimensional of elliptic equations. Bulletin of SamSU. 2014. No. 10 (121). P. 17-25. (in Russian)

8. Mikhlin S. Multidimensional singular integrals and integral equations. Moscow: Fizmatgiz, 1962. (in Russian)

9. Kamke E. Handbook on ordinary differential equations. Moscow: Nauka, 1965. (in Russian) 
10. Beitmen G., Erdeyi A. Higher Transcendental functions. Vol. 2. Moscow: Nauka, 1974. (in Russian)

11. Kolmogorov A., Fomin S. Elements of the theory of functions and functional analysis. Moscow: Nauka, 1976. (in Russian)

12. Tikhonov A., Samarskiy A. Equations Mathematical Physics. Moscow: Nauka, 1977. (in Russian)

13. Smirnov V. The course of higher mathematics. Vol. 4. Part 2. Moscow: Nauka, 1981. (in Russian)

Поступила: 03.06.2019 / Принята: 11.11.2019

\section{КРИТЕРІЙ ОДНОЗНАЧНОЇ РОЗВ'ЯЗНОСТІ СПЕКТРАЛЬНОӦ ЗАДАЧІ ПУАНКАРЕ В ЦИЛІНДРИЧНІЙ ОБЛАСТІ ДЛЯ ОДНОГО КЛАСУ БАГАТОВИМІРНИХ ЕЛІПТИЧНИХ РІВНЯНЬ}

\section{С. А. АЛДАШЕВ}

Інститут математики та математичного моделювання МОН PK, Алмати, Казахстан, E-mail: aldash51@mail.ru

АнотАцІя. Двовимірні спектральні задачі для еліптичних рівнянь добре вивчені, а їх багатовимірні аналоги, наскільки відомо автору, досліджені мало. Це пов'язано з тим, що в разі трьох і більше незалежних змінних виникають труднощі принципового характеру, так як дуже привабливий і зручний метод сингулярних інтегральних рівнянь, що застосовується для двовимірних задач, тут не може бути використаний через відсутність скількинебудь повної теорії багатовимірних сингулярних інтегральних рівнянь. Теорія багатовимірних сферичних функцій, навпаки, досить і повно вивчена. У циліндричної області евклідового простору для одного класу багатовимірних еліптичних рівнянь розглядається спектральна задача Пуанкаре. Розв'язок шукається у вигляді розкладу за багатовимірними сферичними функціями. Доведено теорему існування та єдиності розв'язку. Отримано умови однозначної розв'язності поставленої задачі, які істотно залежать від висоти циліндра.

Ключові словА: багатовимірне еліптичне рівняння, спектральна задача Пуанкаре, багатовимірна циліндрична область, розв'язність, критерій. 